\title{
COMPORTAMENTO DA FREQUÊNCIA CARDÍACA E DA TEMPERATURA CORPÓREA EM COELHOS SUBMETIDOS À PARADA CIRCULATÓRIA CARDÍACA
}

\author{
Daniela Fantini Vale, ${ }^{1}$ Renato Moran Ramos, ${ }^{1}$ Felipp Silveira Ferreira, ${ }^{1}$ Giseli Santos Ferreira, ${ }^{1}$ \\ Fabio Ferreira Queiroz, ${ }^{2}$ Monica Jorge Luz, ${ }^{1}$ Alessandra Castello Costa, ${ }^{1}$ Guilherme Monteiro, ${ }^{3}$ \\ Fernanda Antunes ${ }^{4}$ e Andre Lacerda de Abreu Oliveira ${ }^{4}$ \\ 1. Doutorandos em Ciência Animal pela Universidade Estadual do Norte Fluminense Darcy Ribeiro, UENF. E-mail: danielavale2000@yahoo.com.br \\ 2. Mestrando em Ciência Animal, UENF \\ 3. Médico veterinário autônomo, Clínica Veterinária Canne \& Gatto \\ 4. Professor associado, Laboratório de Clínica e Cirurgia Animal, UENF.
}

\section{RESUMO}

A técnica da parada circulatória total (PTC) ou inflow occlusion consiste em impedir a entrada do sangue às câmaras cardíacas através do clampeamento das veias cavas caudal e cranial e veia ázigos. Contudo, alterações nos parâmetros fisiológicos do paciente podem ser desencadeadas com a aplicação desta técnica. Em enfermidades cardíacas intracavitárias, cuja correção leva alguns minutos, como na estenose pulmonar ou na estenose subaórtica, esta técnica pode ser facilmente utilizada. Por ser de baixo custo e de simples aplicação, é passível de realização em clínicas particulares. Neste ensaio, avaliou-se o comportamento da frequência cardíaca e da temperatura corpórea de coelhos sub- metidos a dois períodos de cinco minutos de PCT, utilizando-se a técnica da inflow occlusion. Um período de recirculação sanguínea entre as oclusões foi realizado a fim de verificar os potenciais benefícios desta prática sobre o comportamento da frequência cardíaca e temperatura corpórea dos animais, parâmetros avaliados ao longo de todo o período transoperatório. Ocorreram dois óbitos, ambos no período de recirculação, dada a ocorrência de fibrilação ventricular. Todavia, com a aplicação do período de recirculação do sangue, foi possível constatar que esta técnica pode ser utilizada em cirurgias intracardíacas, com duração de até dez minutos.

PALAVRAS-CHAVES: Coelhos, inflow occlusion, parada circulatória.

\section{ABSTRACT}

\section{BEHAVIOR OF HEART RATE AND TEMPERATURE IN RABBITS SUBMITTED TO CIRCULATORY ARREST}

The technique of total circulatory arrest (TCA), or inflow occlusion, consists in preventing the blood entry into the heart chambers by clamping the vena cava and the cranial and caudal vena azygos. However, changes in physiological parameters of the patient can be triggered with the application of this technique. This technique can be easily used in heart intra-cavity diseases, whose correction takes a few minutes, as in pulmonary stenosis or subaortic stenosis. Because low cost and simple implementation, it may be carried out in private clinics. In this trial, the behavior of heart rate and body temperature of rabbits submitted to two periods of five minutes of TCA, by the use of inflow occlusion technique, was evaluated. A period of blood recirculation between occlusions was performed to verify the potential benefits of this practice on the behavior of heart rate and body temperature of the animals. These parameters were assessed throughout the perioperative period. There were two deaths, both in the recirculation period due to the occurrence of ventricular fibrillation. Nevertheless, with the implementation of the period of blood recirculation, we verified that this technique can be used in intra-cardiac surgery lasting up to 10 minutes.

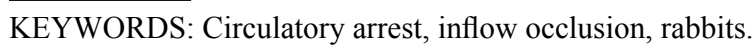




\section{INTRODUÇÃO}

A técnica de oclusão do influxo venoso cardíaco, também chamada estase ou parada circulatória total, foi introduzida em 1951 por VARCO et al., para facilitar os procedimentos cirúrgicos de correção de estenose de valva pulmonar e, subsequentemente, de outras lesões, incluindo estenose de valva aórtica e septectomias atriais (JONAS et al., 1985; SINGH et al., 2006). SWAN (1950), assim como MARTIN \& ESSEX (1951), descreveram experimentalmente em cães a técnica do inflow occlusion e a ligadura da veia ázigos com o objetivo de criar e, posteriormente, corrigir comunicações interatriais (MINGRONE, 2006). HUNT et al. (1992a) realizaram parada circulatória de quatro e oito minutos em cães, que toleraram bem a oclusão, mantendo o ritmo sinusal após procedimento, com uma rápida recuperação hemodinâmica. Em outro momento, o mesmo grupo de pesquisadores procedeu à oclusão apenas da veia cava caudal, observando que a recuperação de todos os parâmetros cardiovasculares ocorreu em apenas cinco minutos após a liberação do fluxo sanguíneo. Esta técnica tornou-se procedimento de eleição nas cirurgias de reparação da veia cava caudal no cão (HUNT et al., 1992b).

Anos depois, a técnica da oclusão do influxo venoso foi realizada durante três minutos em dois cães, sob hipotermia, para correção de cor triatriatum dexter (MITTEN et al., 2001). TOKMAKOGLU et al. (2002) fizeram uso da técnica em questão durante uma miniatriotomia para retirada de trombo no átrio direito

Em medicina humana, a inflow occlusion, juntamente com a hipotermia, teve particular importância na realização de procedimentos intracardíacos até o ano de 1980. Com advento da circulação extracorpórea e seus excelentes resultados, a inflow occlusion é utilizada somente em intervenções cardíacas rápidas ou quando o uso de circulação extracorpórea (CEC) é contraindicado (KIZILTEPE et al., 2003; GARCIA et al., 2009). No entanto, em medicina veterinária, os altos custos e a necessidade de uma equipe treinada dificultam o uso da circulação extracorpórea em cirurgias cardíacas (STOPIGLIA et al., 2001). Esta realidade faz da técnica de oclusão do influxo venoso cardíaco um artifício importantíssimo, tornando praticáveis as intervenções intra e extracavitárias que gerariam um sangramento muito intenso (STOPIGLIA et al., 2001; ODEGARD et al., 2004), tanto no campo da experimentação quanto nas aplicações clínicas (KWASNICKA et al., 2000; FREITAS et al., 2005; GARCIA et al., 2009).

Recentemente, BROCKMAN et al. (2009) realizaram com sucesso o tratamento cirúrgico de uma anomalia congênita rara em um gato de cinco meses, dispondo da oclusão total do fluxo venoso durante dois minutos. Em outro caso, um gato de treze anos foi submetido à mesma técnica, para realizar uma atriotomia direita, a fim de serem retirados alguns parasitas do coração (IIZUKA et al., 2009).

MARTINS et al. (2009) realizaram a ventriculectomia parcial em cães como alternativa cirúrgica ao tratamento da cardiomiopatia dilatada, concluindo que a oclusão do influxo venoso cardíaco é factível, inclusive para procedimentos invasivos, desde que o procedimento não exceda quatro minutos.

A busca por um maior tempo cirúrgico em que se possam realizar as manobras extra e intracardíacas sem os eventuais efeitos nocivos ao paciente é o que incentiva a realização desta pesquisa. Dessa forma, o objetivo deste trabalho é avaliar o comportamento da frequência cardíaca e da temperatura corpórea diante de duas paradas circulatórias cardíacas não consecutivas.

\section{MATERIAL E MÉTODOS}

O presente estudo experimental foi realizado no Laboratório de Cirurgia Experimental Cardiotorácica do Hospital Veterinário da Universidade Estadual do Norte Fluminense Darcy Ribeiro (UENF).

Foram utilizados nove coelhos, adultos, hígidos, de ambos os sexos, pesando entre 2,3 e $3,0 \mathrm{~kg}$, que passaram pela avaliação clínica e laboratorial no momento da admissão (hemograma e bioquímica), a fim de se detectar quaisquer alterações orgânicas que os impediria de participar do estudo. Durante o protocolo experimental, o animal permaneceu sobre um colchão térmico. A aferição da temperatura corpórea foi feita por meio de termometria retal.

Separaram-se os animais em dois grupos experimentais: aqueles alocados no grupo 1 foram submetidos a cinco minutos de oclusão sanguínea e os grupo 2, a uma oclusão adicional por mais cinco minutos. Entre os períodos de parada circulatória, procedeuse a cinco minutos de recirculação sanguínea, como disposto na Tabela 1. 
Idealizaram-se dois grupos experimentais. Todos os animais participaram de ambos os grupos, representando, com isto, o grupo-controle deles mesmos. O primeiro grupo foi representado por uma oclusão sanguínea de cinco minutos. Após um período de recirculação sanguínea de cinco minutos, realizou- se uma oclusão adicional, caracterizando o grupo 2, como disposto na Tabela 1 .

O acompanhamento do comportamento das variáveis fisiológicas frequência cardíaca e temperatura foi realizado de acordo o cronograma representado pela Tabela 1 .

TABELA 1. Momentos de aferição dos parâmetros fisiológicos ao longo do ensaio experimental no Hospital Veterinário da Universidade Estadual do Norte Fluminense Darcy Ribeiro, 2010

\begin{tabular}{|c|c|c|c|c|c|c|}
\hline Grupos & & Grupo 1 & & & Grupo 2 & \\
\hline Momentos & M0 & M2 & M3 & M4 & M5 & M6 \\
\hline Evento & $\begin{array}{c}\text { Plano } \\
\text { anestésico }\end{array}$ & $1^{\circ}$ minuto de $\mathrm{PTC}$ & $\begin{array}{c}5^{\circ} \text { minuto de } \\
\text { PTC }\end{array}$ & $\begin{array}{l}5^{\circ} \text { minuto de recir- } \\
\text { culação }\end{array}$ & $1^{\circ}$ minuto de PTC & $\begin{array}{c}5^{\circ} \text { minuto de } \\
\text { PTC }\end{array}$ \\
\hline $\mathrm{FC}$ & $\mathrm{X}$ & $\mathrm{X}$ & $\mathrm{X}$ & $\mathrm{X}$ & $\mathrm{X}$ & $\mathrm{X}$ \\
\hline $\mathrm{T}^{\circ} \mathrm{C}$ & $\mathrm{X}$ & $X$ & $X$ & $\mathrm{X}$ & $\mathrm{X}$ & $\mathrm{X}$ \\
\hline
\end{tabular}

Legenda: $\mathrm{PTC}=$ parada total circulatória, $\mathrm{FC}=$ frequência cardíaca, $\mathrm{T}^{\circ} \mathrm{C}=$ temperatura .

Os animais foram pré-medicados, pela via muscular, com maleato de acepromazina ${ }^{1}$, na dose de $0,3 \mathrm{mg} \cdot \mathrm{kg}^{-1}$ associado à petidina ${ }^{2}$, na dose de $10 \mathrm{mg}$. $\mathrm{kg}^{-1}$. Vinte minutos após a administração da medicação pré-anestésica realizou-se tricotomia na região lateral do tórax direito, assim como nas faces externas das orelhas para cateterização da veia e artéria auriculares. Durante todo experimento os animais receberam infusão de solução de cloreto de sódio ${ }^{3}$ a $0,9 \%$. Procedeu-se à indução e à manutenção da anestesia com isoflurano ${ }^{4}$ a $1,5 \%$, aproximadamente, CAM $(2,05 \%)$ e oxigênio a $100 \%$ em circuito semiaberto. $\mathrm{O}$ acompanhamento da frequência cardíaca dos coelhos foi feito pelo eletrocardiograma. ${ }^{5}$ Dadas as dificuldades de intubação orotraqueal típica da espécie, optou-se pela realização de uma traqueostomia. $\mathrm{Na}$ linha de incisão, fez-se anestesia infiltrativa com cloridrato de lidocaína ${ }^{6}$ a $2 \%$ com vasoconstritor, na dose de $9 \mathrm{mg} . \mathrm{kg}^{-1}$, realizando-se, na sequência, o acesso cirúrgico da traqueia.

1. Acepran ${ }^{\circledR} 1 \%$, Univet S.A., São Paulo, SP, frasco-ampola contendo $20 \mathrm{ml}$.

2. Dolosal ${ }^{\circledR}$, Cristália, São Paulo, SP, ampola contendo $2 \mathrm{~mL}$ com $1 \mathrm{mg} /$ $\mathrm{mL}$.

3. Cloreto de Sódio ${ }$, São Paulo, SP, frasco de $250 \mathrm{mls}$ a $0,9 \%$.

4. Isoforine ${ }^{\circledR}$, Cristália, São Paulo, SP, frasco com $100 \mathrm{~mL}$.

5 Eletrocardiógrafo TEB ${ }^{\circledR}$ Digital TEB -ECGPC-Tecnologia Eletrônica Brasileira S.A.

6. Xilocaína ${ }^{\circledR, C r i s t a ́ l i a, ~ S a ̃ o ~ P a u l o, ~ S P, ~ a m p o l a ~ c o n t e n d o ~} 20 \mathrm{~mL}$ a $2 \%$.
Colocaram-se os coelhos em decúbito lateral esquerdo, para realização da toracotomia lateral direita, no quarto espaço intercostal.

Uma vez no interior da cavidade pleural, as veias cavas cranial e caudal, bem como a veia ázigos, foram dissecadas em segmentos de $2 \mathrm{~cm}$ a fim de facilitar a sua oclusão pelas pinças vasculares. Imediatamente antes da parada circulatória, os pulmões foram expandidos durante trinta segundos por uma última vez para o esvaziamento das câmaras cardíacas. Após o cessar da ventilação, estabeleceu-se a parada circulatória total inicialmente por um período de cinco minutos, tempo cronometrado a partir da oclusão da veia cava caudal. Na sequência, ocluíram-se as veias ázigos e a veia cava cranial. As referidas veias foram liberadas ao término do período de oclusão de cinco minutos, na sequência inversa à de oclusão.

Em seguida à abertura das pinças, estabeleceu-se um período de cinco minutos de recirculação sanguínea, fato que precedeu o segundo momento de parada circulatória total. A segunda oclusão ocorreu de forma idêntica à primeira, por um período de cinco minutos.

Todos os procedimentos realizados encontramse de acordo com as normas e princípios éticos de experimentação animal estabelecidos pela Comissão de Ética de Uso de Animais (CEUA) da Universidade Estadual do Norte Fluminense Darcy Ribeiro, tendo sido aprovados. 
Na estatística das variáveis fisiológicas, utilizouse a análise de variância (ANOVA) para medidas repetidas, seguida do teste de Tukey, ambos os testes com $95 \%$ de confiabilidade ( $\mathrm{p}<0,05)$. O programa estatístico empregado para esta análise foi o ASSISTAT $^{\circledR}$ versão 7.5 para Windows.

\section{RESULTADOS E DISCUSSÃO}

Os valores referentes à frequência cardíaca e à temperatura corpórea obtidos durante os estudo estão dispostos na Tabela 2.

Os dados apresentados nas Figuras 1 e 2 sobre frequência cardíaca e temperatura foram submetidos à análise estatística por ANOVA e demonstraram que as médias da FC e temperatura variaram significativamente entre si ao longo do experimento. Estes valores são apresentados na Tabela 2.

Todos os animais dos dois grupos experimentais apresentaram no momento zero, já em plano anestésico, batimentos cardíacos e temperatura corpórea retal dentro dos limites fisiológicos para a espécie.

Quanto à frequência cardíaca, especificamente, em ambos os grupos experimentais, os coelhos mantiveram valores dentro do considerado normal para a espécie (Figura 1).

TABELA 2. Valores de $\mathrm{FC}$ e de $\mathrm{T}^{\circ} \mathrm{C}$ (média \pm desvio-padrão) obtidos nos Grupos 1 e 2 durante parada circulatória total pela técnica de inflow occlusion

\begin{tabular}{ccccccc}
\hline & & \multicolumn{2}{c}{ Grupo 1 } & & \multicolumn{2}{c}{ Grupo 2 } \\
Momentos & & Parada de cinco minutos & Parada de cinco minutos adicionais \\
\cline { 2 - 7 } & M0 & M2 & M3 & M4 & M5 & M6 \\
\hline \multirow{2}{*}{ FC } & 231,22 & 200,22 & 213,44 & 217,37 & 211,57 & 171,2 \\
& $\pm 20,49$ & $\pm 38,69$ & $\pm 34,23$ & $\pm 34,36$ & $\pm 35,38$ & $\pm 48,12$ \\
\multirow{2}{*}{$T^{\circ} \mathrm{C}$} & 38,94 & 37,9 & 37,8 & 37,32 & 37,1 & 37 \\
& $\pm 0,83$ & $\pm 0,80$ & $\pm 0,77$ & $\pm 0,90$ & $\pm 0,81$ & $\pm 0,89$ \\
\hline
\end{tabular}

Legenda: $\mathrm{FC}=$ frequência cardíaca, $\mathrm{T}^{\circ} \mathrm{C}=$ temperatura em graus Celsius.

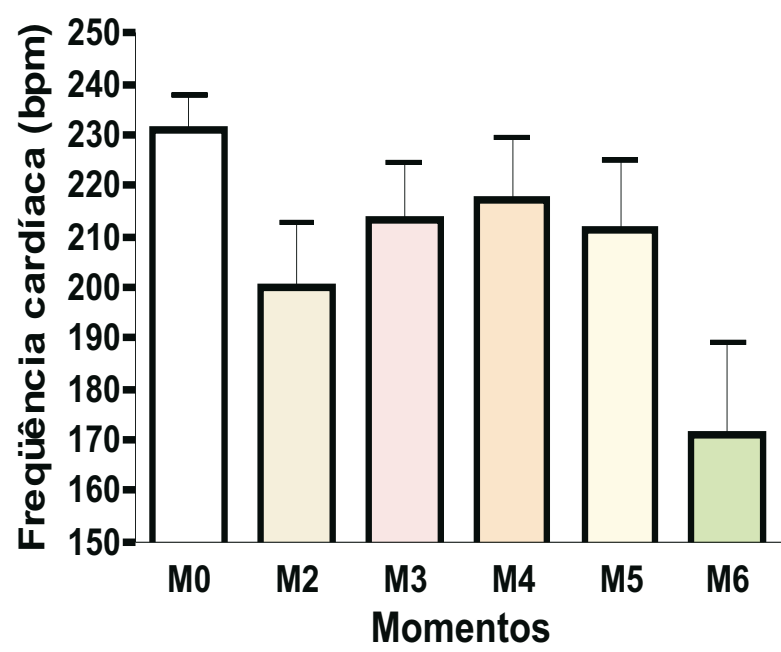

FIGURA 1. Comportamento da média da frequência cardíaca dos coelhos durante as fases do experimento no Hospital Veterinário da Universidade Estadual do Norte Fluminense Darcy Ribeiro, 2010 .
Com o emprego do teste de Tukey, pode-se afirmar que a variação observada pela ANOVA ocorreu de forma não significativa para frequência cardíaca entre a relação das médias dos grupos avaliados neste estudo - M0 e M4; M2 e M5; M3 e M6 (Figura 2). O período de recirculação parece ter seu reflexo positivo neste sentido, mantendo as alterações de frequência no grupo 2 similares àquelas apresentadas no grupo 1,0 que não seria esperado caso não houvesse a recirculação e a parada fosse de dez minutos contínuos. Podese sugerir que a recirculação foi eficiente no sentido de proporcionar a manutenção dos valores de FC no segundo momento de oclusão.

A queda nas médias de M0 e M4 não teve significância. Logo, pode-se inferir que, após cinco minutos de parada circulatória, os animais mantiveram valores de FC semelhantes aos anteriores à oclusão. 
Os valores de referência da frequência cardíaca para os coelhos são bem variáveis, segundo TRANQUILLI et al. (2007) - de 135 a 325 bat/min -, variação que ocorreria pela variedade de tamanhos e raças que compõem a espécie.

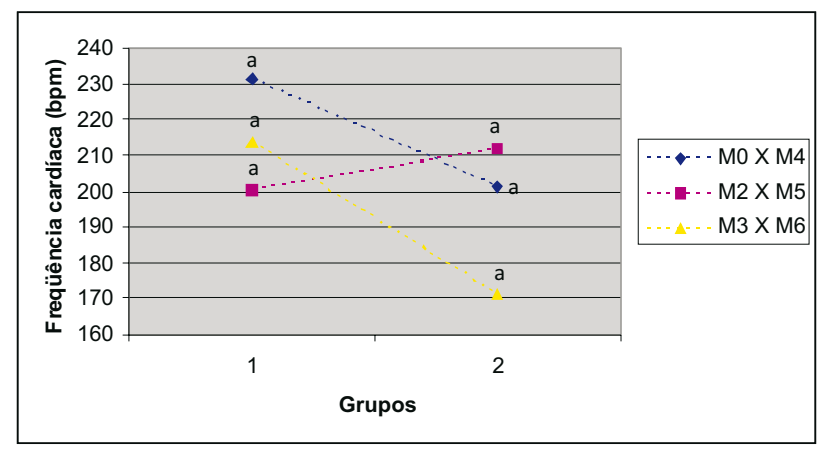

*a/a-não significativo

FIGURA 2. Análise comparativa da frequência cardíaca dos animais entre os grupos 1 e 2. Hospital Veterinário da Universidade Estadual do Norte Fluminense Darcy Ribeiro, 2010.

A ocorrência de taquicardia reflexa é relatada por HUNT et al. (1992b) e GARCIA (2006) em dois momentos durante a realização da técnica de inflow occlusion em cães: no segundo minuto, após o esvaziamento das câmaras cardíacas, e na recirculação sanguínea. No presente trabalho, a ocorrência de taquicardia reflexa não foi observada no momento da oclusão, tampouco no momento de recirculação.

MINGRONE (2006) refere-se a um aumento no valor da FC logo após cinco minutos de recirculação sanguínea no grupo de cães submetidos a oito minutos de parada circulatória. Este fato corrobora o ensaio apresentado, em que a média da FC antes da liberação do fluxo era de $213,44 \mathrm{bat} / \mathrm{min}$ (M3). No entanto, cinco minutos após recirculação foi de 217,37 bat/min (M4). Apesar desse aumento, não houve diferença estatística significativa. A autora ainda cita que no grupo de cães submetidos a sete minutos de parada circulatória o aumento não ocorre, e associa este fato a uma estimulação adrenérgica maior quanto mais longo for o período de estase sanguínea. Comportamento semelhante da FC foi descrito por HUNT et al. (1992b), o que reforça a hipótese aventada por MINGRONE (2006).

MINGRONE (2006) e GARCIA et al. (2009) não avaliaram os cães sob os cinco minutos de parada circulatória. A justificativa se deu pela impossibilida- de de análise dos dados no referido momento, dada a alternância de períodos de taquicardia e bradicardia acarretados pela parada circulatória. Contestando os achados dos citados autores, o presente estudo avaliou o primeiro e o quinto minuto de parada circulatória cardíaca, não sendo observadas taquicardia nem bradicardia, o que pode ser comprovado na análise estatística dos momentos de M0; M2; M3 e M4; M5; M6, em que não houve diferença significativa entre as médias.

No presente trabalho, ocorreram dois óbitos no grupo 2 em M4, sendo o primeiro com um minuto e trinta segundos e o segundo no quinto minuto da recirculação, ambos em decorrência de fibrilação ventricular. Tal fato vem de encontro aos resultados descritos por GARCIA (2006), que observou fibrilação ventricular como causa mortis de dois cães submetidos a oito minutos de inflow occlusion. Nesse ensaio os óbitos dos dois coelhos ocorreram após cinco minutos de parada circulatória cardíaca. É factível correlacionar o óbito do segundo animal no momento M4 com os motivos apresentados por MINGRONE (2006). A autora refere que a ocorrência de fibrilação ventricular durante a realização do inflow occlusion ou logo após a restituição da circulação está relacionada ao quadro de hipóxia do miocárdio ou ao aumento excessivo da estimulação adrenérgica, o que pode ser verificado cinco minutos após a restituição da circulação. A afirmação citada, segundo a autora, ocorre em resposta fisiológica normal à parada circulatória total. Em outros trabalhos o problema da fibrilação também se faz presente. STOPIGLIA et al. (2001) relataram óbito em decorrência de fibrilação ventricular no grupo de cães submetidos a dez minutos de parada circulatória cardíaca. KWASNICKA et al. (2000) também citam um óbito ocorrido no grupo submetido ao mesmo período de oclusão, porém em decorrência de fibrilação atrial. De fato, a fibrilação ventricular é uma das complicações desencadeadas pela parada circulatória sanguínea, encontrada inclusive nesta pesquisa.

Em relação à temperatura corpórea, segundo ORTON et al. (1990), o nível da temperatura do paciente no período transoperatório e o tempo da parada circulatória estão intimamente relacionadas quando da aplicação da técnica do inflow occlusion.

No transoperatório, os coelhos, independentemente do grupo experimental, apresentaram as médias de temperatura corpórea sempre abaixo dos valores fisiológicos. 
Com relação à média da temperatura, os coelhos apresentaram queda durante o procedimento (Figura 3).

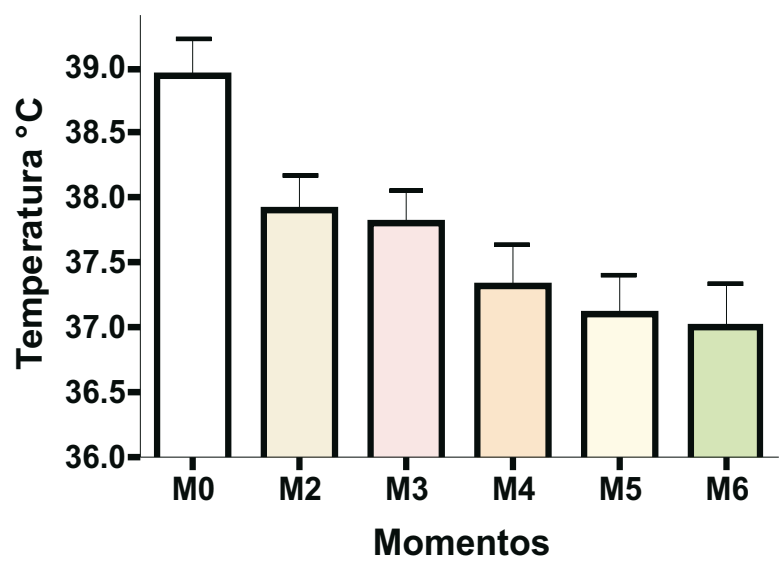

FIGURA 3. Comportamento da média da temperatura dos coelhos durante as diferentes fases do experimento no Hospital Veterinário da Universidade Estadual do Norte Fluminense Darcy Ribeiro, 2010 .

$\mathrm{Na}$ avaliação do comportamento da temperatura corpórea neste ensaio, pôde-se constatar que algumas médias se apresentaram até um grau abaixo do valor para a espécie $\left(38,5^{\circ} \mathrm{C}\right)$, fato que pode ser atribuído ao ineficiente sistema de manutenção térmica realizada à base de colchão térmico. O mesmo pôde ser verificado nos trabalhos de KWASNICKA et al. (2000), STOPIGLIA et al. (2001) e GARCIA et al. (2009), em que o sistema de manutenção térmico utilizado também foi ineficiente para manter a temperatura dos cães dentro dos valores fisiológicos para espécie.

Com o emprego do teste de Tukey, pode-se afirmar que a variação observada pela ANOVA ocorreu de forma significativa para a temperatura entre os momentos M0 e M4. Já entre os momentos M2 e M5 e M3 e M6 não houve diferença estatística significativa (Figura 4).

$\mathrm{Na}$ análise estatística dos momentos M0 e M4, houve diferença significativa nas médias da temperatura apresentadas pelos coelhos. Dessa maneira, podese inferir que a queda da temperatura constituiu-se em um mecanismo de defesa orgânica metabólica contra a parada circulatória, como aventado por GARCIA et al. (2009).

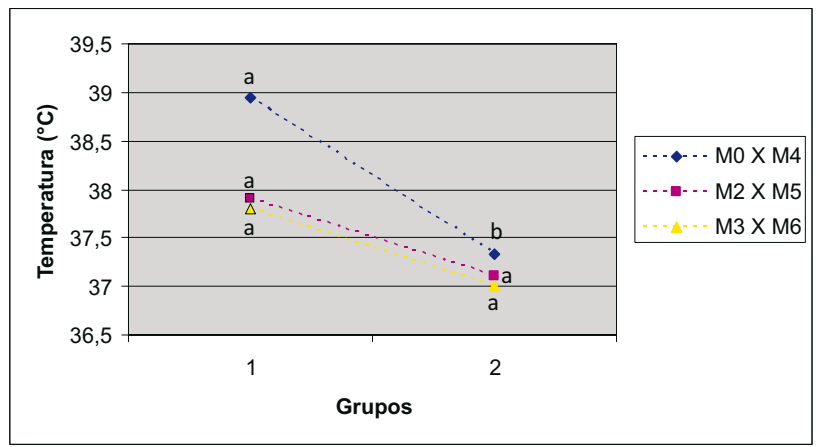

* a/a-não significativo; a/b significativo

FIGURA 4. Análise comparativa da temperatura dos animais entre os Grupos 1 e 2. Hospital Veterinário da Universidade Estadual do Norte Fluminense Darcy Ribeiro, 2010.

Em análise das médias da temperatura apresentadas entre M2 e M5 e M3 e M6, era esperada uma queda maior, principalmente no segundo grupo (M5 e M6), que já havia sofrido os efeitos de uma oclusão anterior, porém a queda da temperatura entre os grupos não foi significativa. Isto corrobora outra hipótese de GARCIA et al. (2009), em que a hipotermia pode ser exclusivamente por conta do processo anestésicocirúrgico e não em decorrência da parada circulatória total. STOPIGLIA et al. (2001) referem queda da temperatura de ambos os grupo experimentais (com cinco e dez minutos de oclusão), também sem diferença estatística significativa entre as médias, o que condiz com os resultados deste trabalho. Estes autores aferem a temperatura dos cães por termometria retal, mesmo método de aferição do presente estudo.

GARCIA (2006) refere que as médias da temperatura apresentadas pelos animais do grupo que sofreu oclusão por oito minutos apresentaram valores superiores às médias dos animais do grupo que sofreram oclusão por sete minutos. Em seu trabalho, GARCIA (2006) afere a temperatura central dos cães. Pela aplicação de diferentes métodos de aferição da temperatura, os resultados deste ensaio não foram compatíveis com os do autor acima citado.

Pode-se ainda discorrer sobre a hipoperfusão periférica causada pela parada circulatória, colaborando, dessa maneira, pela queda da temperatura, já que um dos fatores responsáveis pela manutenção da temperatura é o fluxo sanguíneo periférico. Segundo MINGRONE (2006), quanto menor o fluxo sanguíneo maior a queda da temperatura. 
Segundo LIMA et al. (1996), a queda da temperatura ocorre em virtude da utilização de agentes anestésicos inalatórios. FONSECA et al. (1996) dizem que isoflurano provoca vasodilatação periférica e que, somado ao relaxamento muscular, impede a produção de calor, aumentando a perda por irradiação, condução e evaporação. Esta pode ser mais uma das causas que levaram à queda da temperatura neste trabalho, haja vista a utilização do mesmo fármaco para indução e manutenção da anestesia dos coelhos.

\section{CONCLUSÃO}

O período de cinco minutos de recirculação sanguínea permitiu a manutenção dos parâmetros fisiológicos dos animais compatíveis com a vida, possibilitando um tempo de parada circulatória total de dez minutos e, dessa forma, aumentando a possibilidade de intervenções operatórias intracardíacas em uso da circulação extracorpórea.

A fibrilação ventricular em decorrência de arritmias durante a execução da técnica de inflow occlusion é uma complicação desta técnica, podendo ocasionar o óbito.

\section{AGRADECIMENTOS}

À CAPES, pela bolsa concedida para realização desta pesquisa.

\section{REFERÊNCIAS}

BROCKMAN, D. J.; BORER, K. E.; BAINES, S. J.; HUGHES, D.; FUENTE, V. Partial right ventriculectomy using the incised patch technique to treat double chambered right ventricle and chylothorax in a cat. Veterinary Surgery, v. 38, p. 631-635, 2009.

FONSECA, N. M.; GOLDENBERG, S.; GOMES, P. O.; LIMA, A. P. Anestesia em coelhos. Acta Cirurgica Brasileira, v. 11, n. 2, p. 82-104, 1996.

FREITAS, R. R.; NOGUEIRA, G. A. K. A. ; IRINO, E. T.; SOUZA, S. L.; STOPIGLIA, A. J.; FANTONI, D. T.; BARBUSCI, L. O. D.; LARSSON, M. H. M. A.; JATENE, F. B. Diagnosis and surgical correction of atrial septal defect by inflow occlusion technique. Brazilian Journal of veterinary Animal Science, v. 42, n. 3, p. 193-195, 2005.

GARCIA, D. C. Parada circulatória total em cães por diferentes períodos de tempo: avaliação clínica e hemogasométrica. 2006.93 f. Tese (Mestrado em Clínica Cirúrgica Veterinária) - Universidade de São Paulo, São Paulo, 2006. Disponível em: $<$ http://www.teses. usp.br/teses/disponiveis/10/10137/tde-20032008-115219/>

GARCIA, D. C.; STOPIGLIA, A. J.; MINGRONE, L. E.; FANTONI, D. T. Avaliação clínica de cães submetidos à parada circulatória total por diferentes períodos de tempo através da técnica de inflow occlusion. Pesquisa Veterinária Brasileira, v. 29, n. 2, p. 125-130, 2009.

HUNT, G. B.; MALIK, R.; BELLENGER, C. R.; PEARSO, M. R. B. Total venous inflow occlusion in the normothermic dog: a study of haemodynamic, metabolic and neurological consequences. Research Veterinary Science, v. 52, n. 3, p. 371-377, $1992 \mathrm{a}$.

HUNT, G. B.; MALIK, R.; BELLENGER, C. R.; PERSON, M. R. A new technique for surgery of the caudal vena cava in dogs using partial venous inflow occlusion. Research Veterinary Science, v. 52, n. 3, p. 378-381, 1992 b.

IIZUKA, T.; HOSHI, K.; ISHIDA, Y.; SAKATA, I. Right atriotomy using total venous inflow occlusion for removal of heartworms in a cat. The Journal of Veterinary Medical Science, v. 71, n. 4, p. 489-491, 2009.

JONAS, R. A.; CASTANEDA, A. R.; NORWOOD, W. I.; FREEED M. D. Pulmonary valvotomy under normothermic caval inflow occlusion. Australian and New Zeland Journal of Surgery, v. 55, n. 1, p. 39-44, 1985.

KIZILTEPE, U.; EYILETEN, Z. B.; UYSALEL, A.; AKALIN, $\mathrm{H}$. Acute pulmonary hypertensive crisis after TAPVC repair treated with atrial septectomy with inflow occlusion. International Journal of Cardiology, v. 87, p. 107-109, 2003.

KWASNICKA, K. L.; STOPIGLIA, A. J.; FREITAS, R. R.; FANTONI, D. T. Avaliação hemogasométrica durante a parada circulatória total, inflow occlusion, aplicada por diferentes períodos de tempo em cães sadios. Brazilian Journal of Veterinary Research Animal Science, v. 37, n. 3, p. 234-242, 2000.

LIMA, A. P.; GOLDENBERG, S.; FONSECA, N. M.; NOVO, N. F.; JULIANO, Y. Avaliação de um sistema circular de anestesia, em ventilação mecânica com pressão positiva intermitente: anestesia quantitativa em coelhos (Oryctolagus cuniculus). Acta Cirurgica Brasileira, v. 11, n. 3, p. 108-15, 1996.

MARTINS, L. G. A.; RAISER, A. G.; BRAGA, F. V. A.; RAPPETI, J. C.; POH, V. H. A. Ventriculectomia parcial mediante oclusão do influxo venoso em cães. Ciência Rural, v. 39, p. 1-6, 2009.

MINGRONE, L. E. Avaliação hemodinâmica de cães submetidos à parada circulatória total utilizando a técnica de inflow occlusion. 2006. 122 f. Dissertação (Mestrado em Clínica 
Cirúrgica Veterinária) - Universidade de São Paulo, São Paulo, 2006.Disponível em: <www.teses.usp.br/teses/disponiveis/10/.../ LarissaEckmannMingrone.pdf $>$

MITTEN, R. W.; EDWARDS, G. A.; RISHNIW, M. Diagnosis and management of cor triatriatum dexter in a Pyrenean Mountain dog and an Akita Inu. Australian Veterinary Journal, v. 7, n. 3, p. 177-180, 2001.

ODEGARD, K. C.; SCHURE, A.; SAIKI, Y.; HANSEN, D. D.; JONAS, R. A.; LAUSSEN, P. C. Anesthetic considerations during caval inflow occlusion in children with congenital heart disease. Journal of Cardiothoracic and Vascular Anesthesia, v. 18, n. 2, p. 144-147, 2004.

ORTON, E. C.; BRUECKER, K. A.; MCCRACKEN, T. O. An open patch-graft technique for correction of pulmonic stenosis in the dog. Veterinary Surgery, v. 19, n. 2, p. 148-154, 1990.

SADE, R. M.; CRAWFORD, F. A.; HOHN, A. R. Inflow occlusion for semilunar valve stenosis. Annals Thoracic Surgery, v. 33, n. 6, p. 570-575, 1982.
SINGH, J.; DHALIWAL, R. S.; BISWAL, S.; SWAMI, N. et al. Inflow occlusion in the era of modern cardiac surgery. The Journal of Thoracic and Cardiovascular Surgery, v. 132, n. 5, p. 1246, 2006.

STOPIGLIA, A. J.; FREITAS, R. R.; IRINO, E. T.; POGLIANI, F. C.; SIMÕES, E. A.; KWASNICKA, K. L.; FANTONI, D. T.; JATENE, F. B. Avaliação clínica da parada circulatória total em cães (Canis familiaris). Acta Cirurgica Brasileira, v. 16, n. 4, p. 211-217, 2001.

TOKMAKOGLU, H.; KANDEMIR, O.; GUNAYDIN, S.; YORGANCIOCLU, C.; ZORLUTUNA, Y. Extraction of right atrial thrombus with inflow-occlusion technique in a patient with gastric cancer. Journal of Cardiovascular Surgery, v. 43, p. 899, 2002.

TRANQUILLI, J. W.; THURMON, J. C.; GRIMM, K. A. Lumb \& Jones' veterinary anaesthesia and analgesia. 4. ed. IOWA: Blackwell, 2007. 1096 p.

VARCO, R. L. Temporary vena cavae occlusion for open heart surgery. Surgery, v. 30, p. 42, 1951. 